\title{
Application to Real Power Networks of a Method to Locate Partial Discharges Based on Electromagnetic Time Reversal
}

\author{
A. Ragusa, Member, IEEE, H. Sasse, A. Duffy, Fellow, IEEE, M. Rubinstein, Fellow, IEEE
}

\begin{abstract}
The paper presents an experimental validation of a method to locate partial discharges (PDs) on power distribution and transmission networks. The method is based on electromagnetic time reversal (EMTR) theory, and it uses a Transmission Line Matrix (TLM) model to describe the propagation of the PD signals in the reversed time. Since PDs are regarded as a symptom of insulation degradation, on-line PD location is considered an important approach to monitoring the integrity of a power distribution network, with the aim of detecting and preventing faults and improving network reliability. In this paper, the EMTR-based method is described and its effectiveness in PD localization using only one measurement point is demonstrated in three real $33 \mathrm{kV}$ power lines. Its effectiveness is proved with and without an on-line electromagnetically noisy environment, and its accuracy is evaluated with respect to different signal-to-noise ratio (SNR) levels of the networks. The validation shows that the method is able to locate PDs with an error of $0.14 \%$ with respect to the total length of the line in the absence of noise, and with an error that is always lower than $0.5 \%$ for an SNR down to $-7 \mathrm{~dB}$
\end{abstract}

Index Terms - Cable system, partial discharges, fault prevention, electromagnetic time reversal, power system protection, reliability, TLM modeling.

\section{INTRODUCTION}

P OWER distribution and transmission networks are frequently subject to a range of electromagnetic disturbances arising from both natural and man-made sources. High circulating currents, thermal runaway/overheating, insulation degradation and partial discharges (PD), harmonics, and overvoltages result in poor power quality, supply interruption and outages, often leading to considerable costs. This aggressive environment accelerates cable insulation aging, producing defects on power cables [1]. The insulation failure of

Manuscript submitted May 12, 2021

This work was supported by the European Union's Horizon 2020 Research and Innovation Programme under the Marie Skłodowska-Curie Grant under Agreement 838681. a power cable can have severe social and economic consequences. Statistics indicate that more than $85 \%$ of equipment failures are related to insulation damage [2]. Thus, the adoption of on-line diagnostic methods that can locate insulation degradation is an effective solution for the condition monitoring of power networks to improve their resilience. Insulation deterioration is often caused by PD events. A PD is a localized electrical discharge [3] which starts in a defect of the insulation system of power distribution and transmission lines, and that can produce effects ranging from temporary faults to black-outs. Since PDs are widely regarded as one of the best early warning indicators of insulation degradation [4], the on-line PD location is considered as one of the most suitable methods to perform network integrity assessment and to prevent faults, improving the electricity supply security [5]

The on-line PD location problem in power networks has been widely investigated in the literature. Most on-line methods are based on reflectometry or traveling wave techniques [6]-[14] that use the fact that a PD event produces electromagnetic waves that travel in either direction towards the cable ends. The time of arrival (ToA) methods are the most widely adopted reflectometry-based methods. The incident wave and the reflected waves from the cable ends are measured at different points of the line and the difference in their arrival times allows the PD localization. However, their practical implementation is difficult due to the need for synchronization of the multi-end measured pulses and their accuracy is influenced by the PD signals distortion. Another big challenge is the presence of electromagnetic interference (EMI). On-line EMI can be classified into (i) narrow band noise, that arises from communications systems, (ii) wide band noise that is pulse-type coming from power electronics, due to the increasing use of inverters and VSD/VFD motors on medium voltage networks, lightning and switching operations, and (iii) Gaussian white noise produced by measuring instruments and high voltage equipment [10][11]. PD signals are pulses with a low amplitude, a fast rise time, and a duration of a few hundred nanoseconds. In the network harsh noisy environment, they are

A. Ragusa, H. Sasse, and A. Duffy are with the School of Engineering and Sustainable Development, De Montfort University, Leicester LE1 9BH, UK. (e-mail: antonella.ragusa@dmu.ac.uk; hgs@dmu.ac.uk; apd@dmu.ac.uk).

M. Rubinstein is with the Institute for Information and Communication Technologies, University of Applied Sciences and Arts Western Switzerland, 1401 Yverdon-les-Bains, Switzerland (e-mail: marcos.rubinstein@heig-vd.ch).

“(C) 2021 IEEE. Personal use of this material is permitted. Permission from IEEE must be obtained for all other uses, in any current or future media, including reprinting/ republishing this material for advertising or promotional purposes, creating new collective works, for resale or redistribution to servers or lists, or reuse of any copyrighted component of this work in other works." 
often completely overshadowed by EMI. Wavelet transform (WT) techniques are applied to de-noise PD signals in most detection and location methods [9]-[14]. WT techniques are powerful signal processing tools but they require a considerable computational effort. Yii et al. [10] proposed a multi-end PD location algorithm based on a discrete WT technique for the suppression of white noise. It can locate PDs with errors $<0.05 \%$ but with computational times up to $2500 \mathrm{~s}$. In [13], a PD detection method is proposed that suppresses narrowband noise using a singular value decomposition algorithm and white noise using the empirical WT. The method denoises PD signals with computational times of a few seconds but it distorts the PD wave, affecting the location accuracy; its performance is also reduced for low SNR (signal-to-noise ratio) values.

Therefore, despite the high performance of the existing location methods, their accuracy is still affected by the following factors: 1 . The number of observation points (OPs) versus possible multiple PD location solutions in large and complex networks; 2 . Requirement of a precise time recording for multiple synchronized measurements methods; 3 . Possible loss of GPS signal, used in synchronized measurements methods, impacting the accuracy; 4. De-noising the PD signal. The Electromagnetic Time Reversal (EMTR) theory [15] has been recently used to locate sources of electromagnetic disturbances in power systems. Methods to locate lightning strikes [15] [17] and faults [18][19] have been developed showing a considerable performance improvement with respect to the classical approaches. In [20], a method to locate PDs based on EMTR theory was proposed by the authors. In [20] the method design procedure is given and its effectiveness to locate PDs, using only one OP, is demonstrated in simulations.

In this paper, an experimental validation of the method is presented in real $33 \mathrm{kV}$ power networks. Its robustness against the presence of on-line noise is also proved, showing the method's ability to locate PDs without the need of a PD signal de-noising. These results show the ability of the method to overcome most of the previously described shortcomings associated to the traditional methods. The paper is organized as follows. In Section II the EMTR theory is introduced and its application to the localization of electromagnetic disturbance sources is explained. Section III describes the basic steps of the EMTR PD location method. In Section IV, the experimental validation is presented, the effectiveness of the method in three $33 \mathrm{kV}$ power lines is discussed and its accuracy is evaluated considering, in this first analysis, different SNR levels of white noise superimposed on the PD signals. Finally, concluding remarks are given in Section V.

\section{Electromagnetic Time ReVersal THeOry}

Electromagnetic time reversal (EMTR) methods to locate the sources of electromagnetic disturbances are based on the invariance of Maxwell's equations under time reversal (TR) [15]. Mathematically, TR implies making the substitution $\mathrm{t} \rightarrow-\mathrm{t}$. Consider Maxwell's equations:

$$
\begin{aligned}
& \nabla \cdot(\varepsilon(\vec{r}) \vec{E}(\vec{r}, t))=\rho(\vec{r}, t) \\
& \nabla \cdot(\mu(\vec{r}) \vec{H}(\vec{r}, t))=0
\end{aligned}
$$

$$
\begin{aligned}
& \nabla \times \vec{E}(\vec{r}, t)=-\mu(\vec{r}) \frac{\partial \vec{H}(\vec{r}, t)}{\partial t} \\
& \nabla \times \vec{H}(\vec{r}, t)=\varepsilon(\vec{r}) \frac{\partial \vec{E}(\vec{r}, t)}{\partial t}+\vec{J}(\vec{r}, t)
\end{aligned}
$$

where $\vec{E}$ and $\vec{H}$ are the electric and magnetic fields, $\rho$ is the charge density, $\vec{J}$ is the electric current density, and $\varepsilon$ and $\mu$ are, respectively, the electric permittivity and magnetic permeability.

Applying the time-reversal transformation, the following equations are obtained:

$$
\begin{aligned}
& \nabla \cdot(\varepsilon(\vec{r}) \vec{E}(\vec{r},-t))=\rho(\vec{r},-t) \\
& \nabla \cdot(\mu(\vec{r})(-\vec{H}(\vec{r},-t)))=0 \\
& \nabla \times \vec{E}(\vec{r},-t)=\mu(\vec{r}) \frac{\partial \vec{H}(\vec{r},-t)}{\partial(-t)} \\
& \nabla \times \vec{H}(\vec{r},-t)=\varepsilon(\vec{r}) \frac{\partial \vec{E}(\vec{r},-t)}{\partial(-t)}+(-\vec{J}(\vec{r},-t))
\end{aligned}
$$

Expressions (2) are identical to (1), except for the magnetic field and the current density that have changed sign (see Chapter 1 of [15] for a discussion on the necessity of the sign change).

In essence, the EMTR source location method involves measuring the radiation from the source, time reversing the measured signals, and back-injecting the time-reversed signal. The time reversibility of wave equations and the spatial correlation property of the time-reversal theory allow the refocusing of the time reversed, back-propagated electromagnetic waves into the original disturbance source location. In more detail, when the electromagnetic waves are time reversed and back injected into the original system, they arrive in phase and refocus back to the location of their original source.

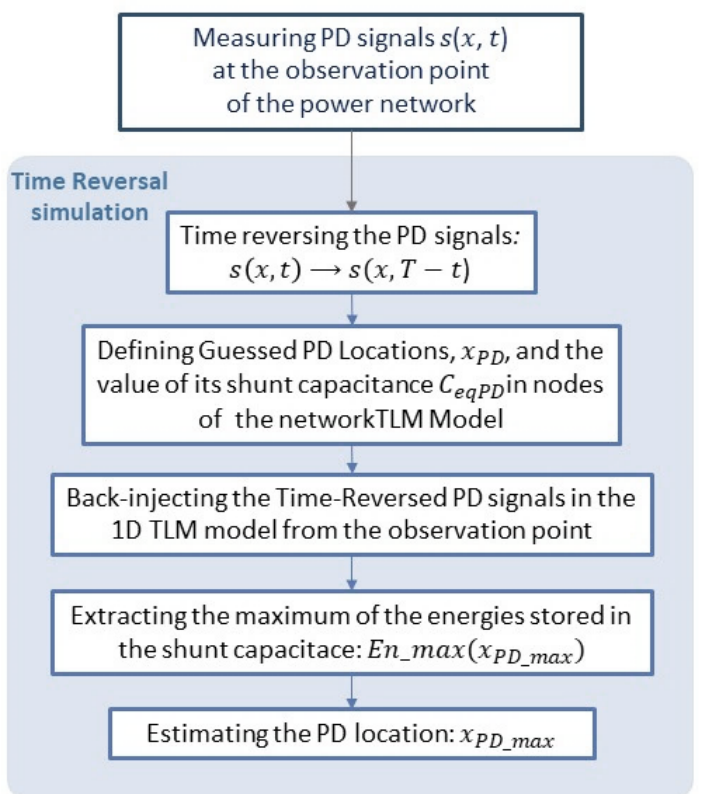

Fig. 1. EMTR method to locate PDs. 


\section{LOCATE PARTIAL DISCHARGES}

The basic steps of the EMTR method to locate PDs [20] in power lines are illustrated in Fig. 1. They are the following:

1. Measuring of the PD-originated signal, $s(x, t)$, at one observation point (OP) along the line.

2. Time reversing the measured PD signal.

3. Defining guessed PD locations (GPDLs) and their shunt impedance in nodes of the 1D Transmission Line Matrix (TLM) model of the network.

4. Simulation of the back-injection of the time-reversed PD signal for different GPDLs using the TLM model.

5. Locating the PD source by identifying the GPDL characterized by the highest energy concentration.

A schematic of the system under analysis is shown in Fig. 2. A power line, formed by a coaxial cable of length $l$, is connected to the load impedances, $Z_{1}$ and $Z_{2}$. The PD event occurs at a given location, $x_{\mathrm{PD}}$ along the line and the PD-generated signal $s(x, t)$ is measured at an OP, selected in this case to be the left end of the line as shown in Fig. 2. The measured signal is time reversed and back injected into a model of the line. Then, several TR simulations are performed to locate the PD source.

The propagation of the PD signal in power distribution and transmission networks is described by the telegrapher's equations, which are time reversal invariant for a nondissipative line [15]. This means that the voltage and current waves, $v(x, t)$, and $i(x, t)$, and their symmetric versions in time, $v(x,-t)$, and $i(x,-t)$, are both solutions of the equations. For nondissipative lines, the telegrapher's equations are:

$$
\begin{aligned}
& \frac{\partial v(x, t)}{\partial x}+L \frac{\partial i(x, t)}{\partial t}=0 \\
& \frac{\partial i(x, t)}{\partial x}+C \frac{\partial v(x, t)}{\partial t}=0
\end{aligned}
$$

where $L$ and $C$ are, respectively, the per unit-length inductance and capacitance of the line, characterized by a propagation speed, $u$, and a characteristic impedance, $Z_{0}$, given by:

$$
u=\frac{1}{\sqrt{L C}} \quad ; \quad Z_{0}=\sqrt{\frac{L}{C}}
$$

Equations (3) are invariant under the TR transformation $(t \rightarrow-t)$ with a change in the sign of the current as follows:

$$
\begin{aligned}
& \frac{\partial v(x,-t)}{\partial x}+L \frac{\partial(-i(x,-t))}{\partial(-t)}=0 \\
& \frac{\partial(-i(x,-t))}{\partial x}+C \frac{\partial(v(x,-t))}{\partial(-t)}=0
\end{aligned}
$$

\section{Observation}

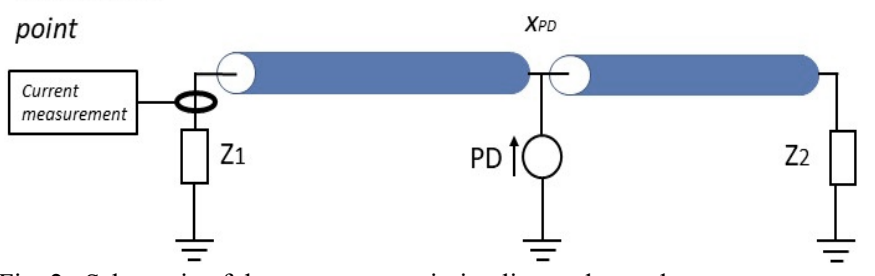

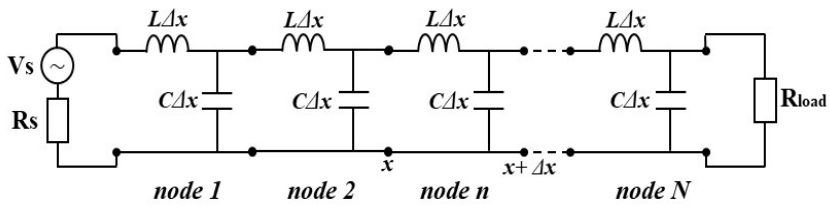

(a)

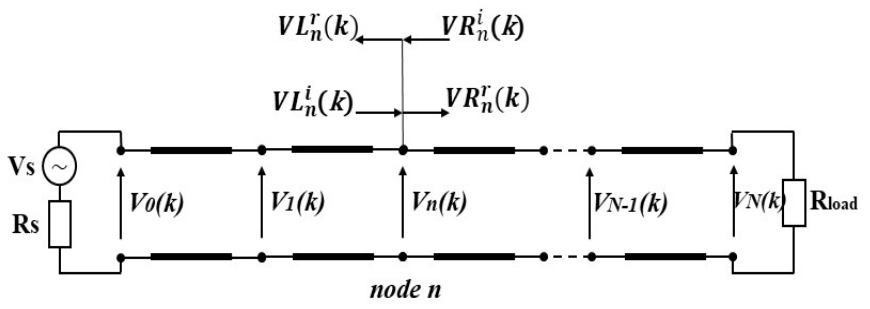

(b)

Fig. 3. The lossless power transmission line (a) and its TLM model (b).

Equations (5), that describe the TR propagation of the PD signals are solved using the TLM method [21], chosen for its flexibility, high efficiency, and its numerical stability. It is a differential-equation-based method, operating in the timedomain, that discretizes the power transmission line, of length $l$, into a series of $N$ segments, of length $\Delta x$, connected as shown in Fig. 3(a). Each $L C$ section of the line is represented by a transmission line of impedance $Z_{0}$, given by relation (4), and characterized by a transit time $\Delta t$ given by:

$$
\Delta t=\frac{\Delta x}{u}=\Delta x \cdot \sqrt{L C}
$$

In the TR simulations, $\Delta t$ is determined by the measurement sampling interval and $\Delta x$ is evaluated by the knowledge of the per-unit-length inductance $L$ and capacitance $C$ of the line, and therefore by the velocity.

Connecting the $N$ sections, the TLM equivalent model of the line is obtained, as shown in Fig. 3(b).

At each node, the voltage pulses, $V_{\mathrm{n}}(k)$, are scattered as they propagate in the lines, generating incident voltages, $V L^{\mathrm{i}}{ }_{\mathrm{n}}(k)$ and $V R_{\mathrm{n}}^{\mathrm{i}}(k)$, and reflected voltages, $V L_{\mathrm{n}}^{\mathrm{r}}(k)$ and $V R_{\mathrm{n}}^{\mathrm{r}}(k)$, respectively on the left and on the right-hand side of the node. Replacing the lines to the right and to the left of the node $n$ by their Thevenin equivalent circuits, and applying Millman's theorem, the voltage $V_{\mathrm{n}}(k)$, and the current $I_{\mathrm{n}}(k)$, at time step $k$, are given by:

$$
\begin{gathered}
V_{n}(k)=\frac{\frac{2 V L_{n}^{i}(k)}{Z_{0}}+\frac{2 V R_{n}^{i}(k)}{Z_{0}}}{\frac{1}{Z_{0}}+\frac{1}{Z_{0}}} \\
I_{n}(k)=\frac{V_{n}(k)-2 V R_{n}^{i}(k)}{Z_{0}}
\end{gathered}
$$

and they are defined by the knowledge of the incident voltages at time step $k$. The incident voltages, at time $k+1$ are evaluated using the condition of the node at time $k$. The incident voltages at node $n$ are, in fact, coincident with the reflected voltages of the nodes $n-1$ and $n+1$ at time $k$.

The PD signal, $s(x, t)$, measured at the OP of the line for an observation period $T$, is time reversed as follows:

Fig. 2. Schematic of the power transmission line under study. 


$$
s(x, t) \rightarrow s(x, T-t)
$$

and it is back injected into the TLM model of the line, from the same OP where it was measured. Then, the TR simulations are performed. The observation period $T$ is defined in order to measure both the direct PD signal and some reflections from the other cable end.

A schematic of the line model used for the TR simulations is shown in Fig. 4.

In each TR simulation, a GPDL is considered in a node of the line model, and the energy stored in the transversal impedance is evaluated. Several TR simulations are performed, changing the position of the GPDL in order to explore the whole line. In the GPDL node, the line shunt impedance is modified to simulate the new shunt capacitive impedance caused by the PD event [20]. The well-known three-capacitor model of PDs [22] and the generalized PD model of Niemeyer [23] were used to define and evaluate the GPDL impedance. This impedance was realized using a stub capacitor at the node, where the voltage, $V_{\mathrm{GPDL}}(k)$, at time $k$, is given by:

$$
V_{G P D L}(k)=\frac{\frac{2 V L_{n}^{i}(k)}{Z_{0}}+\frac{2 V R_{n}^{i}(k)}{Z_{0}}+\frac{2 V c_{n}^{i}(k)}{Z_{p d}}}{\frac{1}{Z_{0}}+\frac{1}{Z_{0}}+\frac{1}{Z_{p d}}}
$$

where $Z_{\mathrm{pd}}$ is the impedance of the stub capacitor in the Thevenin equivalent circuit.

For each TR simulation, the energy, $E_{n}$, stored at the GPDL impedance, normalized with respect to the maximum energy, is evaluated as follows:

$$
E_{n}=\frac{\frac{1}{2} C \sum_{k=1}^{M} V_{G P D L}^{2}(k)}{\frac{1}{2} C \sum_{k=1}^{M} V_{G P D L_{-} m}^{2}(k)}=\frac{\sum_{k=1}^{M} V_{G P D L}^{2}(k)}{\sum_{k=1}^{M} V_{G P D L_{-} m}^{2}(k)}
$$

with $V_{G P D L} m(k)$ being the maximum voltage of the GPDLs, $M=T / \Delta t$ the number of samples and $\Delta t$ the sampling time.

The GPDL characterized by the maximum energy corresponds to the PD location. This is because the pulses of the time reversed PD signal will add up in phase at the real PD location during the backward propagation.

\section{EXPERIMENTAL VALIDATION}

The measurements for the experimental validation of the method were carried out by HVPD Ltd.

The effectiveness of the method was tested on three different $33 \mathrm{kV}$ MV single-phase coaxial cables, respectively Line 1,

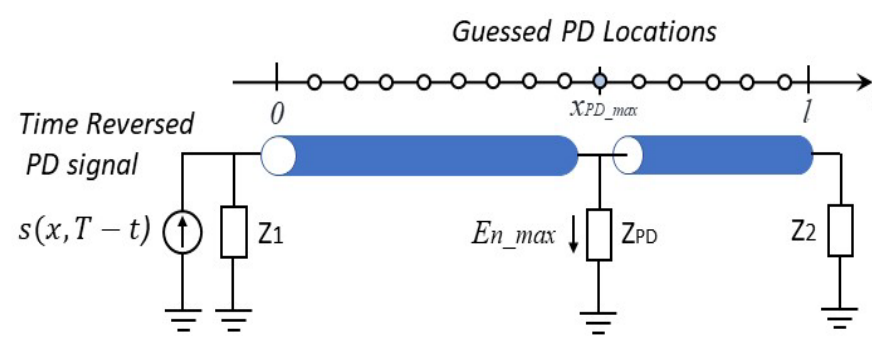

Fig. 4. Schematic representation of the line used for the TR simulations.
Line 2 and Line 3, and in two different cases, the first one without electromagnetic interference (EMI) on the line and the second one with EMI on the line.

The length of the lines under test, the average propagation speed of the cables and the position of the PD source in each line, determined by HVPD using their on-line TDR technology, are reported in Table I.

Fig. 5 shows the measurement set up for each line.

The cables under test were connected to two substations. The impedances at the line ends were unknown. The on-line PD activity was detected with an HFCT (high frequency current transformer) sensor at the Substation 1. The used sensor was an HVPD HFCT $100 / 50$ with a bandwidth of $100 \mathrm{kHz}$ to $20 \mathrm{MHz}$.

Fig. 6 shows the sensor connection at the cable termination. The electromagnetic signals generated by the PD activity, detected by the HFCT sensor, were digitized and processed by

TABLE I

LINE CHARACTERISTICS AND PD SOURCE LOCATIONS

\begin{tabular}{lcc}
\hline \hline \multicolumn{1}{c}{ Line 1 } & Value & PD source \\
\hline Length, $l$ & $1882 \mathrm{~m}$ & \multirow{2}{*}{$475 \mathrm{~m}$} \\
Propagation speed, $u$ & $0.18 \cdot 10^{+9} \mathrm{~m} / \mathrm{s}$ & \\
\hline Line 2 & Value & PD source \\
\hline Length, $l$ & $1900 \mathrm{~m}$ & \multirow{2}{*}{$343 \mathrm{~m}$} \\
Propagation speed, $u$ & $0.18 \cdot 10^{+9} \mathrm{~m} / \mathrm{s}$ & \\
\hline Line 3 & Value & PD source \\
\hline Length, $l$ & $1900 \mathrm{~m}$ & \multirow{2}{*}{$796 \mathrm{~m}$} \\
Propagation speed, $u$ & $0.18 \cdot 10^{+9} \mathrm{~m} / \mathrm{s}$ & \\
\hline \hline
\end{tabular}

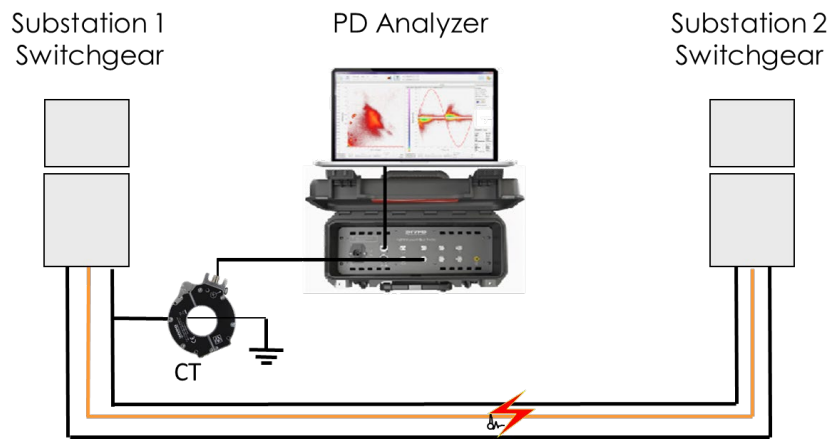

Fig. 5. Measurement set up at HVPD Ltd.

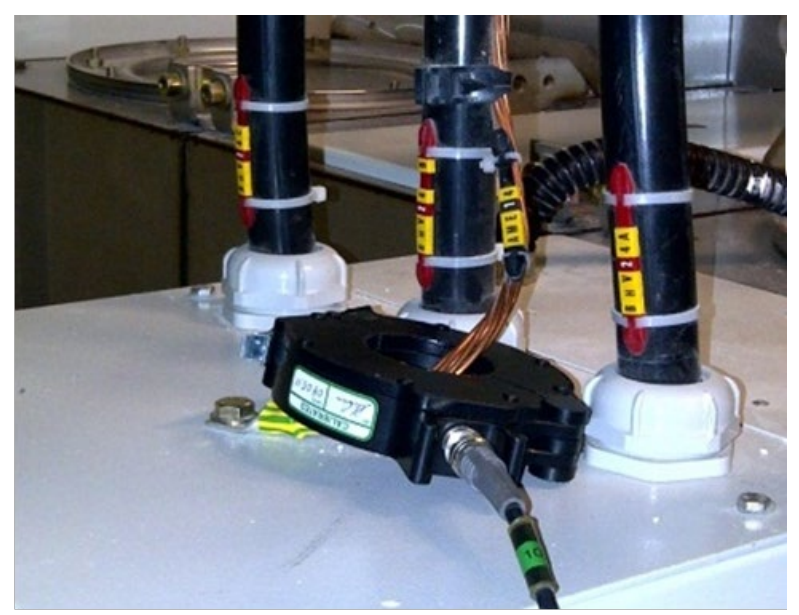

Fig. 6. Measurement of PD signal with HFCT at HVPD Ltd. 


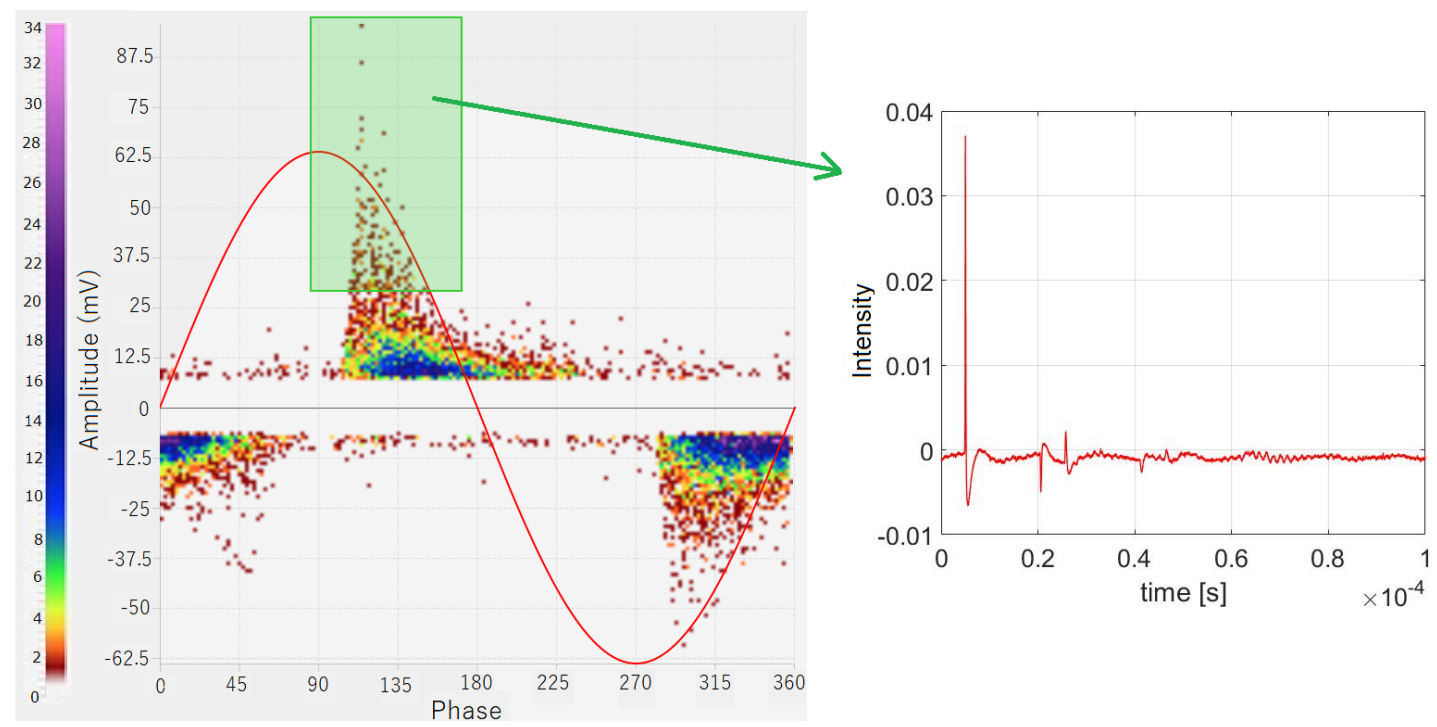

Fig. 7. Amplitude versus Phase PRPD pattern of the PD activity in line 1 evaluated with HVPD Kronos ${ }^{\circledR}$ Ultimate Software.

the HVPD Kronos ${ }^{\circledR}$ Ultimate Software in the PD Analyzer [25], based on the phase-resolved partial discharge (PRPD) analysis method [26].

Fig. 7 presents the PRPD pattern that shows the amplitude versus phase of the PD activity measured on Line 1 . The figure shows, on the right, a PD pulse related to a point within the green window of the pattern.

In order to validate the effectiveness of the proposed location method, the PD source location in each cable was estimated using the EMTR method and the results were compared with the locations reported in Table 1.

To use the EMTR-based method, the characteristic parameters of the 1D TLM model must be defined. Using the available information on the line characteristics reported in Table 1, the parameters of the TLM model were evaluated as follows. A per-unit-length shunt capacitance equal to an average value for $33 \mathrm{kV}$ cables can be considered. In this case a value equal to $C=3.4 \cdot 10^{-10} \mathrm{~F} / \mathrm{m}$ was chosen [21]. Then, the values of the per-unit-length inductance, $L$, and of the characteristic impedance, $Z_{0}$, of the line were evaluated by using relations (4). Finally, a transit time of the TLM model equal to $\Delta t=1 / f_{s}=1 \cdot 10^{-8} \mathrm{~s}$ was used, where $f_{\mathrm{s}}$ is the measurement sampling frequency, equal to $100 \mathrm{MS} / \mathrm{s}$. Hence, from Eq. (6), a space resolution, $\Delta x$, of the TLM model equal to $1.8 \mathrm{~m}$ is obtained. An increase of the sampling frequency allows a reduction of $\Delta x$ but an increase of the computational time to several hours. A value of $1 \cdot 10^{-8} \mathrm{~s}$ has been chosen as a compromise between the location accuracy and the computational effort.

In Table II, the 1D TLM model parameters are reported. The numerical code of the model was developed in MATLAB.

In the following sections, the experimental validation in the

TABLE II

TLM MODEL PARAMETERS

\begin{tabular}{ll}
\hline \hline \multicolumn{1}{c}{ Parameter } & \multicolumn{1}{c}{ Value } \\
\hline Capacitance, $C$ & $3.4 \cdot 10^{-10} \mathrm{~F} / \mathrm{m}$ \\
Inductance, $L$ & $9.077 \cdot 10^{-8} \mathrm{H} / \mathrm{m}$ \\
Characteristic impedance, $Z_{0}$ & $16.34 \Omega$ \\
Transit time, $\Delta t$ & $1 \cdot 10^{-8} \mathrm{~s}$ \\
\hline \hline
\end{tabular}

two cases, with and without EMI on the line, is described.

\section{A. Validation in the case without EMI on the line}

The PD signal was measured on Line 1 at Substation 1 of Fig. 5 , at the left end of the line.

The measured PD current signal is presented in Fig. 7. As can be seen, the measured signal is characterized by alternating positive and negative pulses, suggesting that the line terminations are characterized by negative reflection coefficients. Hence, $Z_{1}$ and $Z_{2}$ were set to a value much smaller than $Z_{0}$ in the TLM model (namely $0.1 \Omega$ ). Even if the cable was connected directly to a power transformer, a low value for the input impedance is reasonable for such high frequency transients [27]. The measured PD signal was time reversed, according to relation (8), as shown in Fig. 8, and then injected into the TLM model of the line. Since the direct signal and the reflected waves were in the time range $0-5 \mathrm{~ms}$, only this portion of the signal was used for the TR simulations to reduce the computational time. Several TR simulations were performed, changing the position of the GPDL along the line, in order to locate the PD source. A first scan of the line was carried out choosing GPDLs $10 \mathrm{~m}$ apart from each other. After that, a refined search was performed, reducing the distance between the GPDLs to $1.8 \mathrm{~m}$ only in the section of the line where the first scanning revealed the maximum concentration of the energy associated to the time reversed signals propagation.

Fig. 9 shows the results of the location method. As the figure shows, using a step of $10 \mathrm{~m}$ between each GPDL, the method identifies the maximum energy around a location at $479 \mathrm{~m}$ from the left end of the cable. The computational time of the first line scan was about 2 minutes. Then, reducing the step to $1.8 \mathrm{~m}$, the search was repeated in the line section around the $479 \mathrm{~m}$ location, in the range $450-500 \mathrm{~m}$ from the left end of the line, and the PD source was located at $478 \mathrm{~m}$. The computational time in this case was $15 \mathrm{~s}$. As Fig. 9 shows, the method locates the PD with a difference of less than $3 \mathrm{~m}$ with respect to the location shown in Table 1, hence with an error of about $0.14 \%$.

\section{B. Validation in the case of EMI on the line}

The effectiveness of the method was also tested for a noisy environment in the power network. 

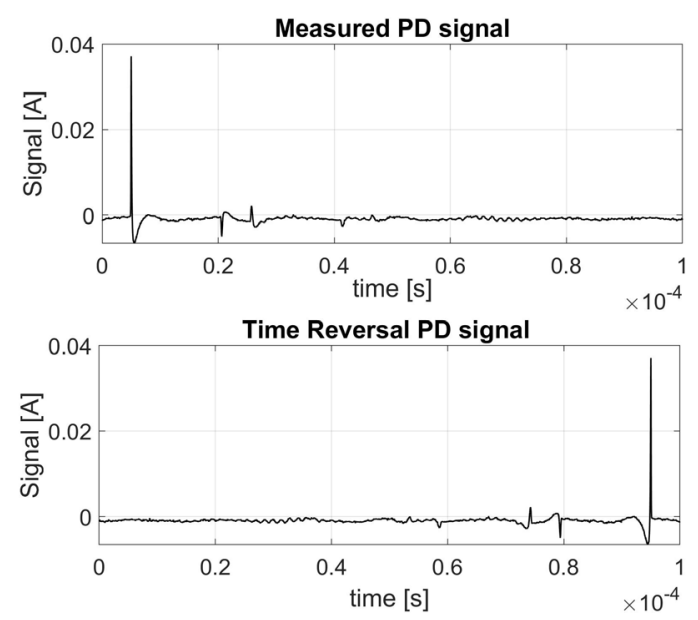

Fig. 8. Time reversed PD signal measured in line 1.

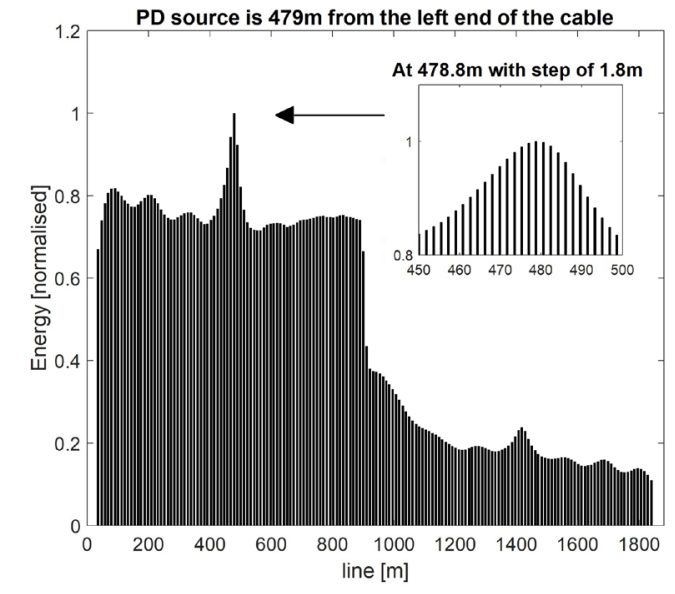

Fig. 9. Normalized energy at the GPDLs in line 1.

Fig. 10 shows the PD signal that was measured at the left end of Line 2, where the PD source was $343 \mathrm{~m}$ from the left end of the cable. The signal is affected by the line noise, but the reflected pulses can be quite easily distinguished.

Applying the method following the previously adopted procedure, the results shown in Fig. 11 were obtained.

The method, using a step size of $10 \mathrm{~m}$ between the GPDLs, showed the highest energy concentration around a location $338 \mathrm{~m}$ from the OP, and it localized the PD at $340 \mathrm{~m}$ when the experiment was repeated using a step size of $1.8 \mathrm{~m}$ in a section

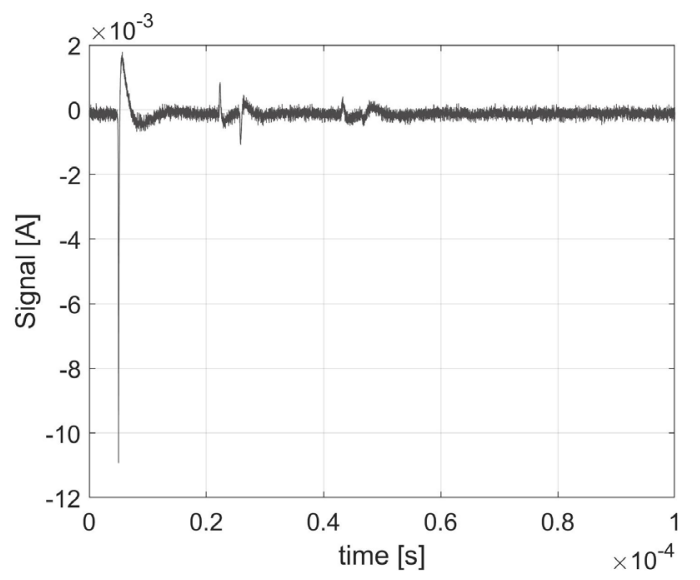

Fig. 10. PD signal measured in Line 2.

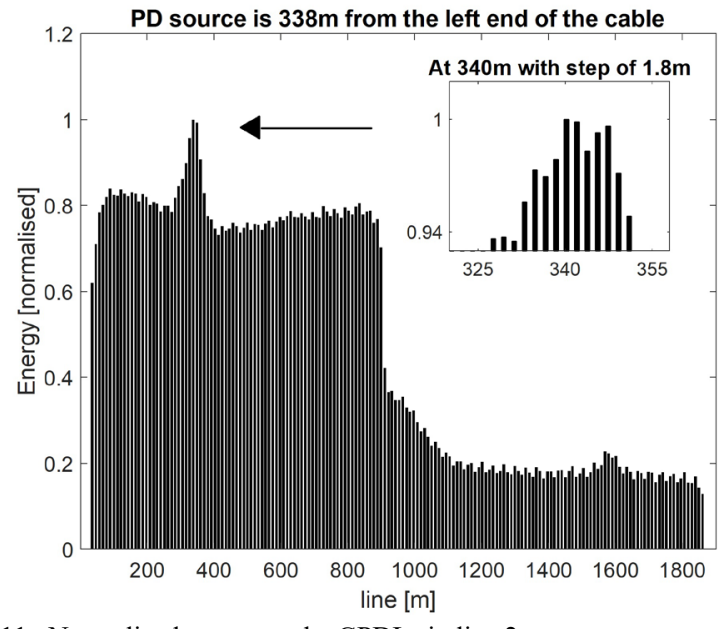

Fig. 11. Normalized energy at the GPDLs in line 2.

of the line around the $338 \mathrm{~m}$ location. The identified location is close to the real one with an error of $0.15 \%$.

Fig. 12 shows the PD signal measured at the left end of line 3 , where the PD source was $796 \mathrm{~m}$ from the left end of the cable. The signal is strongly affected by the line noise and only the direct signal and the first two reflections can be distinguished.

Applying the EMTR-based method to localize the PD source, the results shown in Fig. 13 were obtained. The method, using

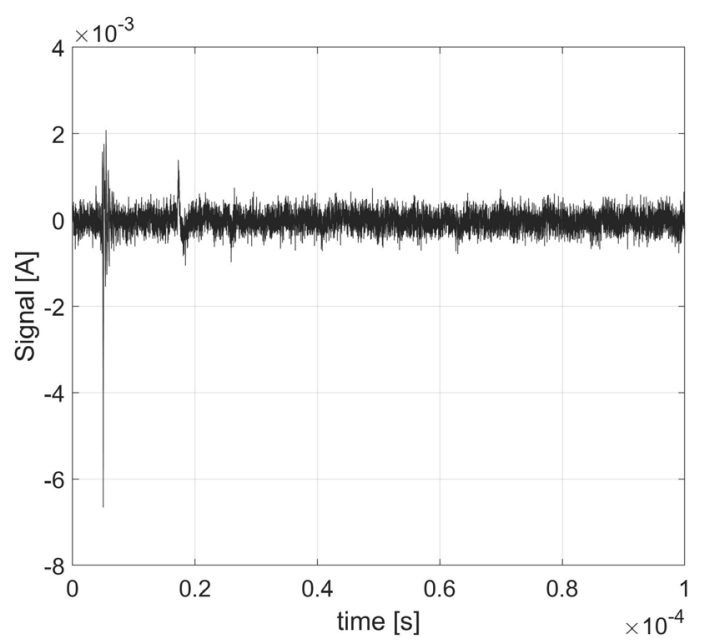

Fig. 12. PD signal measured at the $\mathrm{OP}$ in line 3.

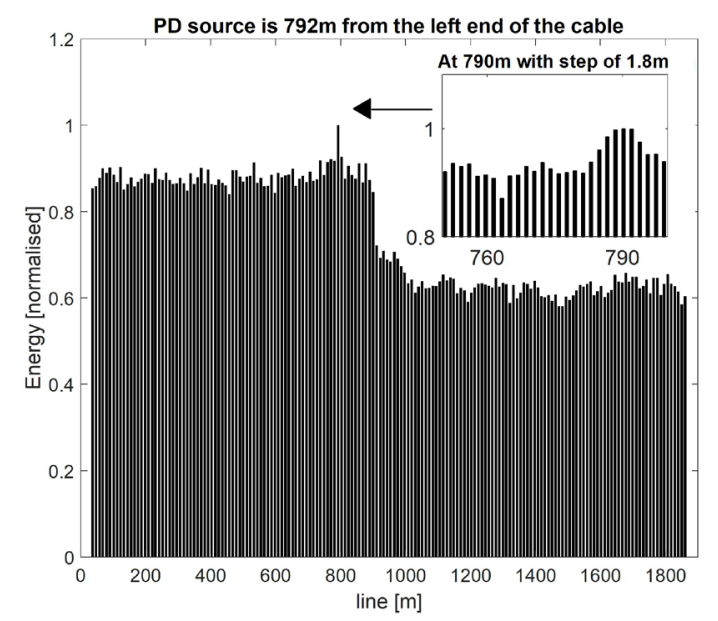

Fig. 13. Normalized energy at the GPDLs in line 3. 
a step size of $10 \mathrm{~m}$ between the GPDLs, showed the highest energy concentration around $792 \mathrm{~m}$ from the OP, and it localized the PD at $790.2 \mathrm{~m}$ when the experiment was repeated using a step size of $1.8 \mathrm{~m}$ in a section of the line around the 792 $\mathrm{m}$ location. The identified location is close to the location reported in Table 1 with an error of $0.31 \%$.

Fig. 14 shows, in the frequency domain, the PD signal measured on Line 3. As the figure shows, the EMI superimposed on the signal is white noise plus a narrowband component at $35 \mathrm{MHz}$.

To test the accuracy of the method with different noise levels on the network, a Gaussian white noise, leading to several levels of SNR (signal-to-noise ratio), was added to the EMI-free PD signal measured on Line 1, and, for each SNR, the effectiveness of the method was evaluated. In order to analyze the worst EMI conditions, negative values of SNR were considered, corresponding to noise with a power higher than the PD signal power. The white Gaussian noise was added artificially in MATLAB.

In Fig. 15, the PD signal with the added white noise with three different levels of SNR are presented.

Fig. 16 and 17 show the results of the localization method when the SNR is, respectively, $-2 \mathrm{~dB}$ and $-4 \mathrm{~dB}$. A step of $10 \mathrm{~m}$ is used for the first scan of the line when the $\mathrm{SNR}=-2 \mathrm{~dB}$ and

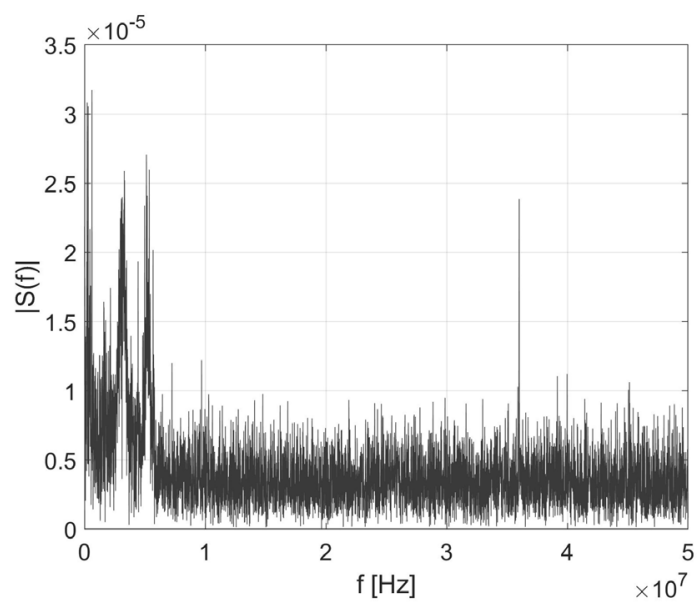

Fig. 14. Frequency spectrum of the PD signal measured in line 3.
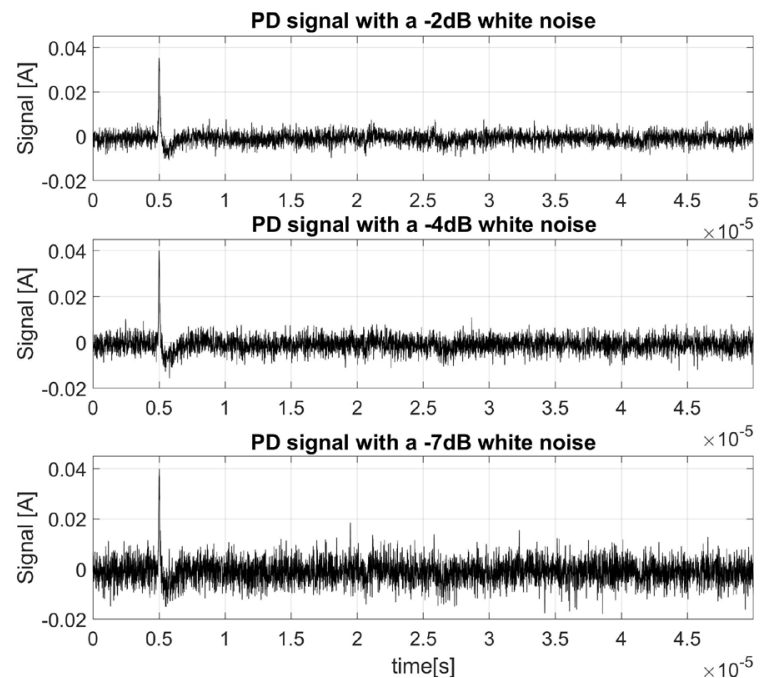

Fig. 15. Measured PD signal with added white noise corresponding, respectively, to $-2 \mathrm{~dB},-4 \mathrm{~dB}$ and $-7 \mathrm{~dB}$ SNR.

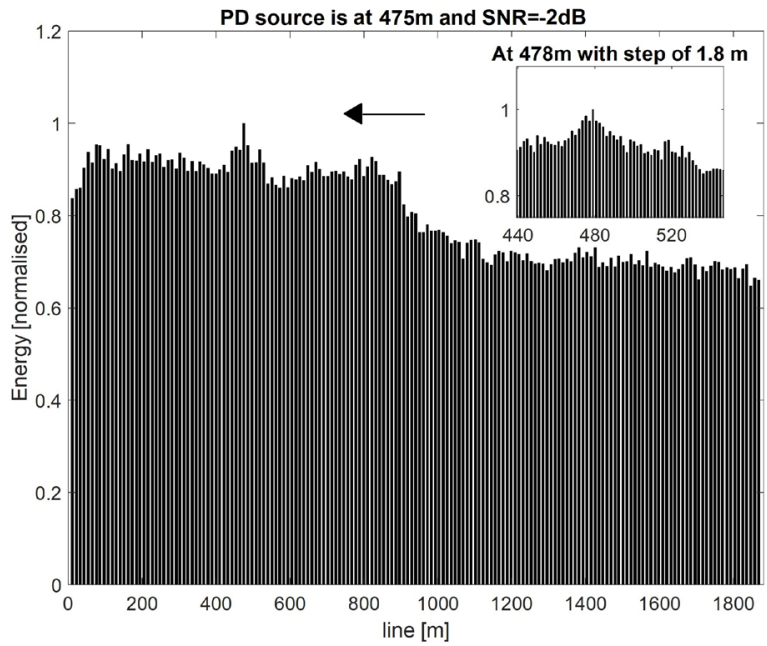

Fig. 16. Results of the localization method with a $\mathrm{SNR}=-2 \mathrm{~dB}$.

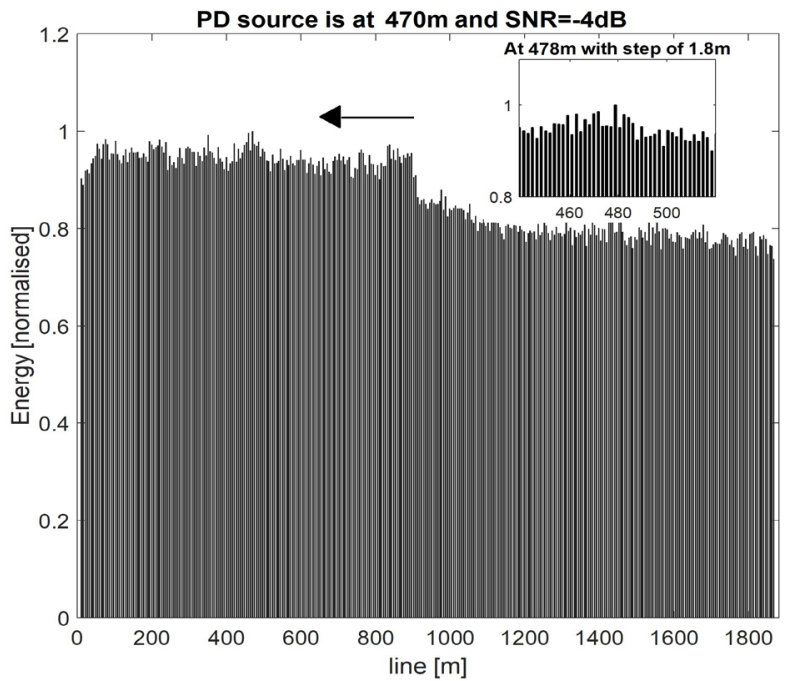

Fig. 17. Results of the localization method with a $\mathrm{SNR}=-4 \mathrm{~dB}$.

a step of $5 \mathrm{~m}$ with an $\mathrm{SNR}=-4 \mathrm{~dB}$. The step reduction to $5 \mathrm{~m}$ implies an increase of the computational time of $30 \mathrm{~s}$. As the figures show, the method is able to localize the PD source even with a high noise level.

The method was tested reducing the value of the SNR to find the lowest level that leads to the failure of the method.

Fig. 18 shows the localization error (evaluated with respect to the locations reported in Table I) versus the tested SNR values.

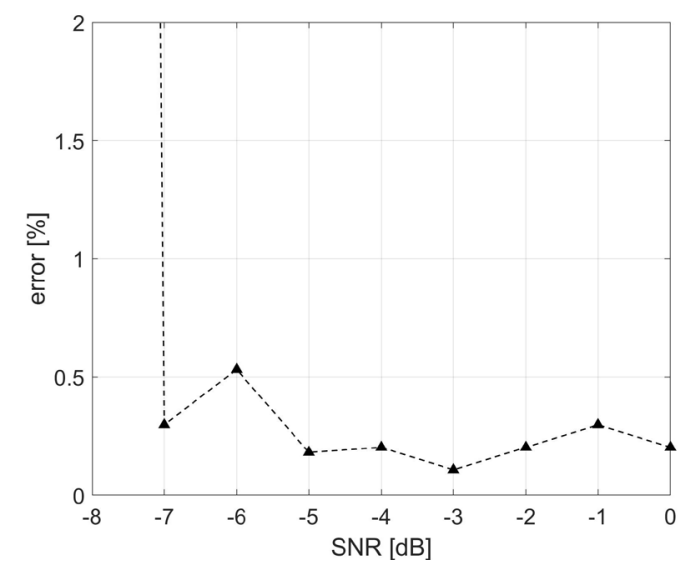

Fig. 18. Error in PD localization versus SNR. 
As the figure shows, the method can locate the PD source with an error $<0.5 \%$ with SNR values down to $-7 \mathrm{~dB}$.

The above results show that the EMTR performance in terms of PD location accuracy and robustness against noise is very promising. Furthermore, the implementation of the method is quite straightforward, and it does not require the complex computational efforts of the WT-based location methods[8].

\section{CONCLUSION}

The experimental validation of a new method to locate partial discharge events on power networks based on the electromagnetic time reversal theory and on the use of a 1D TLM model for the time reversed simulation was described in this paper. The effectiveness of the method was illustrated on three $33 \mathrm{kV}$ MV lines and with different SNR levels. The validation procedure showed that the method is able to locate PDs, with a computational time of less than 3 minutes and an error of about $0.2 \%$ for an SNR level as low as $-5 \mathrm{~dB}$. The error remains lower than $0.5 \%$ for an SNR between $-5 \mathrm{~dB}$ and $-7 \mathrm{~dB}$, with respect to the PD location evaluated with the existing technology. Moreover, the paper shows that the method can locate the PD sources with good accuracy using only the knowledge of the line length and the PD signal's propagation speed. A further improvement in the localization accuracy can be obtained if the propagation speed of the PD signal and of the line characteristics and impedances at the line ends are available. In our future investigations, an error sensitivity analysis with respect to the level of knowledge of the line parameters and of the impedance at the line terminations will be developed, together with the experimental investigation of the method effectiveness also in branched networks with homogeneous and inhomogeneous line sections and different types of EMI on the lines.

\section{ACKNOWLEDGMENT}

The authors would like to extend their thanks for the leadership on, and many constructive discussions about, electromagnetic time reversal by Professor Farhad Rachid, from the École Polytechnique Fédérale de Lausanne, Switzerland and for the support from HVPD Ltd, particularly M. Foxall, M. Seltzer-Grant and A. Polley, who provided the measurements used in figures 8,10 and 12 .

\section{REFERENCES}

[1] F. Auzanneau, "Wire Troubleshooting and diagnosis: Review and perspectives" - Progress in Electromagnetics Research B, Vol. 49, 2013.

[2] S. Refaat, M. Sham - "A Review of Partial Discharge Detection, Diagnosis Techniques in High Voltage Power Cables" - IEEE Int. Conf. on Comp. Pow. Elect. and Power Eng., 2018.

[3] EN 60270 - High-voltage test techniques - Partial discharge measurements (IEC 60270:2000) - 2001.

[4] Z. Du, P. K. Willett, M. S. Mashikian - "Performance Limits of PD Location Based on Time-domain Reflectometry"- IEEE Trans. on Diel. and El. Ins., Vol. 4, No. 2, April 1997.

[5] G. Fulli, F. Profumo, and E. Bompard, Electricity Security in the EU: Features and Prospects, Joint Res. Centre, Brussels, Belgium, Aug. 2018

[6] M. S. Mashikian, R. Bansal, R. B. Northrop - "Location and Characterization of Partial Discharge sites in Shielded Power cables" IEEE Trans. on Pow. Del., Vol. 5, No.2, April 1990.

[7] F. P. Mohamed, W. H. Siew, J. J. Soraghan, S. M. Strachan - "Partial Discharge Location in Power Cables using a Double Ended Method
Based on Time Triggering with GPS"- IEEE Trans. on Diel. and El. Ins., Vol. 20, No. 6; Dec. 2013.

[8] W. He, Q. Wang, C. Huang, H. Li, D. Liang, "A cost-effective technique for PD testing of MV cables under combined $\mathrm{AC}$ and damped $\mathrm{AC}$ voltage", IEEE Trans. Power Del., vol. 33, no. 4, Aug. 2018.

[9] G. Robles, M. Shafiq, J. M. Martínez-Tarifa, "Multiple Partial Discharge Source Localization in Power Cables Through Power Spectral Separation and Time-Domain Reflectometry", IEEE Trans, on Instrumentation and Measurement, Vol. 68, No. 12, Dec. 2019.

[10] C. C. Yii, M. N. K. H. Rohani, M. Isa and S. I. S. Hassan- "Multi-end PD Location Algorithm using Segmented Correlation and Trimmed Mean Data Filtering Techniques for MV Underground Cables" - IEEE Trans. on Diel. and El, Ins. Vol. 24, No. 1; Feb. 2017.

[11] H. Zhang, T.R. Blackburn, B.T. Phung, D. Sen - "A Novel Wavelet Transform Technique for On-line Partial Discharge Measurements Part 1: WT De-noising Algorithm"- IEEE Trans. on Diel. and El. Ins., Vol. 14, No. 1; Feb. 2007.

[12] A. M. Gaouda, A. El-Hag, T.K. Abdel-Galil, M.M. Salama, R. Bartnikas, "On-Line Detection and Measurement of Partial Discharge Signals in a Noisy Environment"- IEEE Transactions on Diel. and El. Insul. Vol. 15, No. 4; August 2008.Pgs. 1162-1773.

[13] J. Zhon, X.Bi, Q. Shu, , M. Chen, D. Zhou, D. Zhang, "Partial Discharge Signal Denoising Based on Singular Value Decomposition and Empirical Wavelet Transform" IEEE Trans, On Instrumentation and Measurement, Vol. 69, No. 11, Nov. 2020.

[14] S. Zhou, J. Tang, C. Pan, Y. Luo, K. Yan, "Partial Discharge Signal Denoising Based on Wavelet Pair and Block Thresholding", IEEE Access, June 30, 2020.

[15] F. Rachidi, M. Rubinstein, M. Paolone, "Electromagnetic Time Reversal - Application to Electromagnetic Compatibility and Power System" John Wiley \& Sons Ltd, 2017; pp. 95-97.

[16] G. Lugrin, N. Mora, F. Rachidi, M. Rubinstein, G. Diendorfer, "On the Location of Lightning Discharges Using Time Reversal of Electromagnetic Fields", IEEE Trans. on EMC, Vol. 56, No. 1, 2014.

[17] H. Karami, M. Azadifar, A. Mostajabi, M. Rubinstein, F. Rachidi, "Numerical and Experimental Validation of Electromagnetic Time Reversal for Geolocation of Lightning Strikes" IEEE Tran. on EMC, Vol. 62, No. 5, Oct. 2020.

[18] S.Y. He, Y.Z. Xie, Z. Wang, F. Rachidi, B.Y. Liu, Q. Li, X. Kong, "Norm Criteria in the Electromagnetic Time Reversal Technique for Fault Location in Transmission Lines", IEEE Trans. on EMC, Vol. 60, No. 5, Oct. 2018.

[19] Reza Razzaghi, Gaspard Lugrin, Hossein Manesh, Carlos Romero, Mario Paolone, and Farhad Rachidi - An Efficient Method Based on the Electromagnetic Time Reversal to Locate Faults in Power Networks IEEE Transactions on Power Delivery, Vol. 28, No. 3, July 2013.

[20] A. Ragusa, H. Sasse, A. Duffy, F. Rachidi, M. Rubinstein "Electromagnetic Time Reversal Method to Locate Partial Discharges in Power Networks using 1D TLM modelling" - IEEE Letters on EMC Practice and Applications, Vol.3, No 1, March 2021.

[21] C. Christopoulos, The transmission-line modeling method - TLM, Institute of Electrical and Electronics Engineers, 1995.

[22] A. Ragusa, H. Sasse, A. Duffy, Towards Modeling Partial Discharge Phenomena and Propagation in Power Networks Using the Transmission Line Matrix Method, Energies 2021, 14(3), 689; 29 Jan. 2021.

[23] L. Niemeyer, "A generalized approach to partial discharge modeling" IEEE Trans. on Diel. and El. Ins., vol. 2, no. 4, pp. 510-528, Aug. 1995

[24] https://www.elandcables.com/electrical-cable-and-accessories/cablesby-standard/dno-approved-cable. Last access 10 May 2021.

[25] L. Renforth, M. Seltzer-Grant, R. Mackinlay, S. Goodfellow, D. Clark, R. Shuttleworth, "Experiences from over 15 Years of on-line partial discharge (OLPD) Testing of in-service MV and HV cables, switchgear, transformers and rotating machines", IEEE IX Latin American Rob. Sym. and Colombian Conf. on Aut. Conf., Bogota, Colombia, 1-4 Oct. 2011. 
[26] R. Altenburger, C. Heitz, and J, Timmer, "Analysis of phase-resolved partial discharge patterns of voids based on a stochastic process approach", J. Phys. D: Appl. Phys. 35 (2002) 1149-1163.

[27] F.M. Tesche, P.R. Barnes, The HEMP response of a power distribution line, IEEE Trans. on Power Delivery, Vol. 4, Nr. 3, 1989.

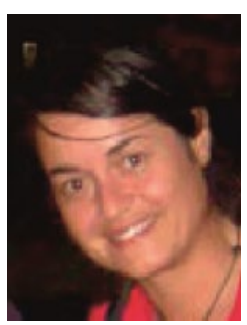

Antonella Ragusa, (M'08) received the Master and Ph.D. degrees in Electrical Engineering from the University of Palermo, Italy, in 2001 and 2006, respectively. In 2007, she worked at FIAT Group Automobiles (Turin) within the group of Engineering and Design. She has been a permanent researcher at the Institute of Marine Engineering (INM) of National Research Council (CNR) of Italy, Palermo since 2008. Currently, she is a Marie Curie Research Fellow (MSCA-IF) at De Montfort University of Leicester, UK. She serves as reviewer for IEEE International Conferences and journals. Her research interests include electromagnetic compatibility, computational electromagnetics and smart grids.

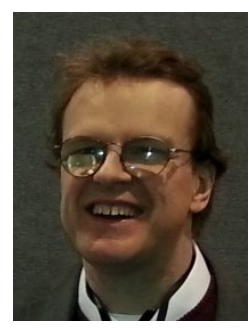

Hugh G. Sasse, received the B.Sc. (Hons) degree in electronic engineering from the University of York, York, U.K., in 1985, and has received his $\mathrm{PhD}$. degree in 2010 from De Montfort University, Leicester, U.K. His research is on optimization of physical layer components for communications systems at De Montfort University.

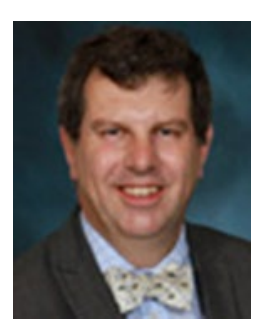

Alistair Duffy, (SM'04, F'14), is Professor of Electromagnetics and Director of the Institute of Engineering Sciences at De Montfort University (DMU), Leicester, UK. $\mathrm{He}$ received his BEng (Hons) and MEng degrees in 1988 and 1989, respectively, from University College, Cardiff, University of Wales. He read for his $\mathrm{PhD}$ with professors Christopoulos and Benson at Nottingham University, graduating in 1993. He also holds an MBA from the Open University, UK, graduating in 2004. He was awarded his DSc from Cardiff University in 2019 for his body of research on the validation of computational electromagnetics. He is a Fellow of the IEEE and President of the IEEE EMC Society. He has published approximately 300 papers, mostly on his research interests of validation of computational electromagnetics; physical layer components, particularly communications cabling, and electromagnetic compatibility testing.

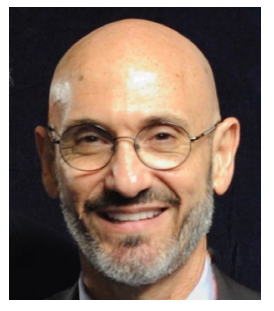

Marcos Rubinstein (M'84-SM'11-F'14) received the Master's and Ph.D. degrees in electrical engineering from the University of Florida, Gainesville, USA. After a postdoctoral fellowship at the Swiss Federal Institute of Technology in Lausanne, where he worked in the fields of electromagnetic compatibility and lightning, he moved to Swisscom as a project engineer, and then program manager in charge of projects in lightning, numerical electromagnetics and EMC. In 2001, he moved to the University of Applied Sciences and Arts of Western Switzerland HES-SO, Yverdon-les-Bains, where he is currently a full Professor, head of the Advanced Communication Technologies Group and a member of the IICT Institute Team. $\mathrm{He}$ is the author or coauthor of more than 300 scientific publications in reviewed journals and international conferences. He is also the coauthor of seven book chapters and one of the editors of a book on Electromagnetic Time Reversal. He served as the Editor-in-Chief of the Open Atmospheric Science Journal and he currently serves as an Associate Editor of the IEEE Transactions on Electromagnetic Compatibility. He is presently the President of the Swiss National Committee of the International Union of Radio Science (URSI).

Prof. Rubinstein received the best Master's Thesis award from the University of Florida. He received the IEEE achievement award and he is a co-recipient of the NASA's Recognition for Innovative Technological Work award. He is also the recipient of the ICLP Karl Berger Award. He is a Fellow of the IEEE and of the SUMMA Foundation, a member of the Swiss Academy of Sciences and of the International Union of Radio Science. 